\title{
Development and Validation of a Measure for Metacognitive Factors in Body Dysmorphic Disorder Patients
}

\author{
Masoud Nikfarjam', Mehdi Rabiei2 ${ }^{*}$, Vahid Donyavi ${ }^{3}$, Hassan Zareei Mahmoodabadi ${ }^{4}$ \\ ${ }^{1}$ Department of Psychiatry, Shahrekord University of Medical Science, Shahrekord, Iran \\ ${ }^{2}$ Department of Clinical Psychology, Baqiyatallah University of Medical Sciences, Tehran, Iran \\ ${ }^{3}$ Departments of Psychiatry, AJA University of Medical Science, Tehran, Iran \\ ${ }^{4}$ Departments of Psychology, Yazd University, Yazd, Iran \\ Email: *rabiei psychology@yahoo.com
}

Received 11 April 2015; accepted 19 May 2015; published 22 May 2015

Copyright (C) 2015 by authors and Scientific Research Publishing Inc.

This work is licensed under the Creative Commons Attribution International License (CC BY).

http://creativecommons.org/licenses/by/4.0/

(c) (i) Open Access

\begin{abstract}
Background and Objectives: Recent studies explored the relationships between metacognitive beliefs and body dysmorphic disorder. The purpose of this study was to develop and validate one self-report scale of metacognitive factor about body dysmorphic. Methods: Development of this scale involved three steps: 1) items generated from a qualitative study with lecture and care professionals, 2) expert panel review, and 3) pilot test. A factor analysis of the responses from 600 participants suggested the creation of a 25 -item scale. Results: Study showed that scores on the 25-item measure correlated with five theoretically related constructs, including positive and negative metacognitive beliefs about body dysmorphic, thought-fusion and metacognitive control strategies. Convergent validity of Body Dysmorphic Metacognitive Scale (BDMCS) with Yale-Brown Obsessive Compulsive Scale was modified for Body Dysmorphic Disorder (BDD) and Thought Fusion Instrument $(\mathrm{r}=0.44, \mathrm{r}=0.52, \mathrm{P}<0.001)$. The factors were able to determine 83.14 percent of the variance. The Cronbach's alpha for the five factors was above 0.83 . Additionally, results have been shown to possess good psychometric properties. Conclusions: The scales may prompt future research into BDD and facilitate clinical assessment and case formulation.
\end{abstract}

\section{Keywords}

Body Dysmorphic Disorder, Metacognition, Metacognitive Beliefs about Body Dysmorphic, Self-Report Scale

\footnotetext{
${ }^{*}$ Corresponding author.
} 


\section{Introduction}

Body dysmorphic disorder (BDD; previously called dysmorphophobia) is characterized by a preoccupation with an imagined defect in individual's appearance or, exaggeration of a slight physical abnormality [1]. The DSM-5 currently is going to consider grouping these disorders in a new diagnostic category: obsessive-compulsive and related disorders [2]. This categorization would involve the inclusion of obsessive-compulsive, hoarding disorder, body dysmorphic, trichotillomania, and pathological skin picking [3]. A recent Dutch study found that $3 \%$ to $8 \%$ of the patients in dermatology and plastic surgery clinics of an academic hospital are suffering from BDD [4]. Psychological and pharmacological treatments for BDD have received increasing attention in the past 10 years. Although psychological and pharmacological treatment approaches for BDD have been evaluated, the relative effectiveness of these two types of interventions has not been examined. Wiliams et al. [5] conducted a meta-analysis of randomized clinical trials and case series studies involving psychological (i.e., behavioural, cognitive-behavioural, cognitive) or medication therapies. Their findings support the effectiveness of both types of therapy, but suggest that cognitive-behavioural treatment (CBT) may be the most useful in the long term. Also, Rabiei et al. [6] reported that Metacognitive therapy (MCT) is an effective treatment for BDD, with effects being somewhat more pronounced on Thought-Fusion symptoms than on BDD symptoms. MCT deals with the way patients with BDD think and it assumes that the problem rests with inflexible and recurrent styles of thinking in response to negative thoughts, feelings and beliefs. In the Rabiei et al. [6] study, patients were taught that metacognitive beliefs, such as the belief that worry or rumination is an effective desirable coping strategy, are important factors contributing to the maintenance of BDD.

Metacognition refers to the psychological structures, beliefs, events and processes that are involved in the control, modification and interpretation of thoughts [7] [8]. All the above constructs interact in maintaining maladaptive cognition and behavior in the metacognitive conceptualization of psychological dysfunction [9]. The Self-Regulatory Executive function (S-REF) [9] theory was the first to conceptualize the role of metacognition in the etiology of psychological disturbances. In this theory, Wells and Matthews [9] argue that a common style of thinking across psychological disorders leads to dysfunction. They propose that psychological disturbance is maintained by a combination of perseverative thinking styles, maladaptive attentional routines, and dysfunctional behaviors. The S-REF theory has led to the development of disorder-specific models of depression [10], generalized anxiety disorder [9], obsessive-compulsive disorder [8], and post-traumatic stress disorder (Wells, 2000). Metacognitive beliefs have been found to be positively associated with many psychological disorders such as depression [10] obsessive-compulsive symptoms [11], body dysmorphic disorder [6] [12]. Also, Cooper and Osman [13] suggested that patients with BDD engage in metacognitive processing in relation to their concerns with appearance. In their study, patients reported attempts to distract themselves from their (invariably distressing) images, which had few advantages and many disadvantages. Images increased self-consciousness and decreased self-confidence. Some patients sought to counter their distress by recalling past positive memories. All patients made negative self-judgments as a result of having these images. Thus, as suggested by Veale [14], metacognition may be an important feature of information processing in BDD and one of the ways in which the symptoms of the disorder are suppressed. Therefore, theoretically it can be a beneficial angel to incorporate from a cognitive perspective to BDD. Further research is needed into the phenomena of metacognition, including its characteristics, functions, and role in the maintenance of the distressing symptoms of BDD. Imagery has been accorded to have a particularly important role in the maintenance of BDD, where mental self-images are thought to be a particularly central feature of a cognitive conceptualization [14].

There are many examples of metacognitive knowledge, including beliefs concerning the significance of particular types of thoughts (e.g. "Having thought about dysmorphic appearance means I am dysmorphic") and positive metacognitive beliefs about emotional self-regulation, mirror checking, grooming, emotions (e.g. "I need to control my anxiety at all times"), and beliefs about cognitive competence (e.g. "I do not trust my problem-solving capabilities”).

Following the S-REF model, the cognitive attentional syndrome (CAS) is a feature for many disorders [12]. There are clear evidences of CAS in BDD (e.g. worry \& rumination, threat monitoring, mirror gazing and mirror checking, and coping behaviors that backfire). Thus, a treatment that would decrease CAS and metacognition associated with it should be effective for BDD [12].

Rabiei et al. [6] reported that Metacognitive therapy (MCT) is an effective treatment for BDD. Furthermore, the effect was more pronounced in Thought-Fusion symptoms than in BDD symptoms. MCT deals with the way 
patients with BDD think and it assumes that the problem rests with inflexible and recurrent styles of thinking in response to negative thoughts, feelings and beliefs. In their study, the patients were taught that metacognitive beliefs, such as believing rumination is an effective coping strategy, are important factors contributing to the maintenance of BDD [6].

Although these studies have provided some basis for possible relation between metacognition and body dysmorphic disorder, there is still some problems in diagnosing and assessing body dysmorphic disorder and a need for a standard assessment tool to measure special metacognition dimensions of body dysmorphic disorder.

The main aim of this study was to develop a body dysmorphic metacognition scale and to determine its validity (construct and convergent), reliability and to compare metacognitive factors in patients with BDD and normal peoples.

\section{Material and Method}

The overall aim of this study was to develop and evaluate select psychometric properties of a self-report measure of Body Dysmorphic Metacognitive (BDMCS) assessing metacognitive factors in people with body dysmorphic disorder.

\subsection{Participants}

The study was performed in 2014. The samples consisted of 600 peoples (280 males 320 and females) from city of Isfahan, Iran. Participants age ranged from 18 to 40 years $(M=25.5$; S.D. $=2.4)$.

\section{Measure}

Development of this scale involved three steps: 1) items generated from a qualitative study with lecture and care professionals, 2) expert panel review, and 3) pilot test.

In-depth interviews were conducted with 10 treatment professionals (metacognitive therapists, body dysmorphic disorder professionals). We aimed to generate the minimum number of items. To ensure content validity, the first two authors, metacognitive therapists, body dysmorphic disorder professionals, thoroughly discussed the above-mentioned themes and generated 25 items to develop the Body Dysmorphic Metacognitive Scale. This first version was sent for comments to an expert panel of two experienced body dysmorphic disorder. They commented on the content of the items as valid and relevant. In phase 2, the initial testing and refinement of the Body Dysmorphic Metacognitive Scale was conducted by a pilot study. Forty undergraduate and graduate students were invited to complete a questionnaire comprising the Body Dysmorphic Metacognitive Scale and scales validating it. The Body Dysmorphic Metacognitive Scale showed good reliability, with Cronbach's alpha of 0.91 .

The measuring tools in this study were: Body Dysmorphic Metacognition Scale, Thought Fusion Instrument and Yale-Brown Obsessive Compulsive Scale Modified for BDD.

Body Dysmorphic Metacognitive Scale: this measure consisted of 25 items used to assess metacognitive errors or distortions related to body dysmorphic disorder. In order to obtain adequate face validity, a pool of items used to construct BDMS from the data obtained in an earlier study [6] and transcripts of therapy sessions. Responses to each item were required on a 4-point rating scale as follow: 1 (do not agree), 2 (agree slightly), 3 (agree moderately) and 4 (agree very much).

Yale-Brown Obsessive Compulsive Scale Modified for Body Dysmorphic Disorder_(BDD-YBOCS; [15]: This is a reliable and valid 12-item semi-structured clinician administered instrument that evaluates current BDD severity. It assesses BDD-related preoccupations, repetitive behaviors, insight, and avoidance [16]. The reliability and validity of the BDD-YBOCS translated version was demonstrated previously by Rabiei et al. [17] in both healthy and patient subjects. They showed that alpha coefficients ranged from 0.78 to 0.93 for BDD-YBOCS total score and for its subscales (preoccupations and repetitive behaviors).

Thought Fusion Instrument (TFI) [18]: This is a 14-item self-report scale that assesses metacognitive beliefs about the meaning, significance and dangerousness of intrusive thoughts. It consists of items tapping thoughtaction fusion (TAF), thought-event fusion (TEF) and thought-object fusion (TOF). Each item is rated on a 0 100 scale (where " 0 " represents "I do not believe this at all", and " 100 " represents "I am completely convinced that this thought is true”). There are limited psychometric data available, but Gwilliam et al. obtained excellent 
reliability (Cronbach's alpha is 0.89 ) for this instrument. The scale appears to have a single factor structure combining all items, and preliminary evidence supports its convergent and discriminate validity [18]. Moreover, Khoramdel et al. [19] showed that the translated version of TFI in both non-patients and clinical samples had proper reliability and validity.

\subsection{Data Analysis}

In order to examine the factor structure of Body Dysmorphic Metacognition Scale (BDMCS) we conducted both exploratory and confirmatory factor analysis. For this analysis, the Structural Equation Modeling program AMOS 5 was used. values of the Goodness of Fit Index (GFI) and the Adjusted Goodness of Fit Index (AGFI) close to 1 represents a good fit, values of the Root Mean Square Residual (RMR) and Standardized Root Mean Square Residual (SRMR) below 0.05 represents a good fit, and values less than 0.08 represents an acceptable fit. The Kaiser-Meyer-Olkin measure of sampling adequacy index was 0.93 and Bartlett's test of sphericity was significant $(\mathrm{p}<0.001)$ indicating that the sample was appropriate for factor analysis. Absolute skewness and kurtosis values were close to 0 indicating variables were univariate normal. Item-total correlation values were all greater than 0.30 indicting acceptability of the items [20]. Due to the relatively small sample size (i.e., less than 10 participants per item) the more stringent criteria recommended by Floyd and Widaman [21] was used requiring factor loadings to be greater than .40 in order for items to be retained.

\section{Results}

Table 1 provides general characteristics of the study sample:

\begin{tabular}{|c|c|c|}
\hline \multirow[b]{2}{*}{ Gender (n) } & Female & 320 \\
\hline & & 280 \\
\hline \multirow[b]{2}{*}{ Age (yr) } & Mean age (S.D.) & $25.5(2.4)$ \\
\hline & Min-max & $18-40$ \\
\hline \multirow{5}{*}{ Occupational status (n) } & Working full-time & 166 \\
\hline & Student & 256 \\
\hline & Part-time work & 70 \\
\hline & Unemployed & 93 \\
\hline & Retired & 15 \\
\hline \multirow{3}{*}{ Education (n) } & Primary school & 90 \\
\hline & High school & 406 \\
\hline & University & 104 \\
\hline \multirow{4}{*}{ Psychotropic medication (n) } & SSRI & 30 \\
\hline & SNRI & 35 \\
\hline & Benzodiazepines & 100 \\
\hline & Antipsychotics & 15 \\
\hline \multirow{2}{*}{ Psychiatry disease duration (yr) } & Mean length (S.D.) & $3(12)$ \\
\hline & Min-max & $0-15$ \\
\hline
\end{tabular}




\subsection{Scale Validity}

Item-total correlation values were all greater than 0.30 indicting acceptability of the items [20]. A principal components method of factor extraction was performed on data from BDMCS and a five-factor solution (Eigen values of 5.76, 1.78, 1.51, 1.07, and 0.769 respectively) based on a screen test which was subjected to oblique rotation.

Oblique rotation was selected because we expected factors to be correlated. The identified five factors (accounting for $83.8 \%$ of variance) reflected the following domains:

\subsection{Metacognition Control Strategies}

1) I lose most of my energy, when thoughts regarding my dysmorphic body surround.

2) I am worried and sad with concerns regarding my dysmorphic body.

3) I keep thinking to my image until I finally obtain a result.

4) Most of time I use mental energy for my appearance. \{This sentence is vague: Most of time, when I use energy it is a mental energy (rather than other types of energy) OR I use metal energy for my appearance most of my time in a day\}

5) Most of time I focus on my thoughts and don't pay attention on my around. (I am occupied by my thoughts most of the time rather than paying attention to my environment or surroundings.)

\subsection{Though-Fusion}

1) I accept my thoughts (about dysmorphic appearance) after they rise.

2) I believe in my thoughts (about dysmorphic appearance) as a reality.

3) I don't challenge mythoughts (about dysmorphic appearance)

4) I think my thoughts and images (about dysmorphic appearance) are fact.

5) When these thoughts and images (about dysmorphic appearance) arise in my mind, I look at the mirror and verify these thoughts.

\subsection{Positive Metacognitive Beliefs about Emotional Self-Regulation}

1) Mirror checking, grooming, camouflaging makes me more affectionate. (I feel more affectionate by mirror checking, grooming and camouflage or avoidance).

2) Mirror checking, grooming, camouflaging makes me more confident. (I feel more confident by mirror checking, grooming and camouflage).

3) Mirror checking, grooming, camouflaging makes me feel more relaxed. (I feel more relaxed by mirror checking, grooming and camouflage).

4) Mirror checking, grooming, camouflaging reduce my anxious feelings. (I feel less anxious by mirror checking, grooming and camouflage).

5) Mirror checking, grooming, camouflaging makes me more sociable. (I feel more relaxed by mirror checking, grooming and camouflage).

\subsection{Positive Metacognitive Beliefs about Cognitive Self-Regulation}

1) Mirror checking, grooming, camouflaging makes me think more clearly. (I think more clear by mirror checking, grooming and camouflage).

2) Mirror checking, grooming, camouflaging help me to control my thoughts. (I control my thoughts by mirror checking, grooming and camouflage).

3) Mirror checking, grooming, camouflaging make my negative thoughts more bearable. (I tolerate my negative thoughts easily by mirror checking, grooming and camouflage).

4) Mirror checking, grooming, camouflaging help me focus my mind (I am more focused by mirror checking, grooming and camouflage).

5) These thoughts (body dismorphic) make me more being aware of my appearance. (I am more aware of my appearance by these thoughts.) 


\subsection{Negative Metacognitive Beliefs about Cognitive Harm and Uncontrollability}

1) I have no control over my mirror checking, grooming, camouflaging.

2) My mirror checking, grooming, camouflaging persists no matter how I try to control it. (I have no control over my mirror checking, grooming and camouflaging habits)

3) If I cannot control my mirror checking, grooming, camouflaging will make me crazy.

4) Mirror checking, grooming, camouflaging will damage my mind.

5) Mirror checking, grooming, camouflaging will make me to lose my control

\subsection{The BDMCS Structure}

The fit of the five-factor model that emerged in the exploratory factor analysis in sample was evaluated with confirmatory factor analyses. The fit of this model was good according to all fit indices $\left(x^{2}=21.12, \mathrm{df}=34, \mathrm{p}>\right.$ 0.05 ; RMSEA $=0.001$; CFI $=0.99$ ). Thus, the results from the confirmatory factor analysis indicated the correlated five-factor model provided representation for the factor structure of BDMCS. These findings, reported in Table 2, demonstrated that the model had overall fit to the data. The model had best fit to the data and was closely related to the theoretical assumptions of the BDMCS.

\subsection{Convergent Validity}

The convergent validity was supported by testing correlation between BDMCS and Yale-Brown Obsessive Compulsive Scale modified for BDD and TFI. BDMCS and its subscales were positively associated with Yale-Brown Obsessive Compulsive Scale modified for BDD $(\mathrm{p}<0.001)$. Table 3 shows correlation results between the total score of scale and its factors after factor analysis.

\subsection{Internal Consistency}

Cronbach's alpha coefficients of the total score were generally high, indicating a high degree of homogeneity. The internal consistencies of the subscales were moderate to high, with Cronbach's alpha values ranging from 0.81 to 0.91 (see Table 4).

\section{Discussion}

Extrapolating from Wells and Matthews' [9] metacognitive theory of emotional disorders, and recent evidence supporting the existence of specific metacognitive beliefs about dysmorphic body in patients with BDD by Cooper and Osman [13], we conducted this study, aiming at developing and validating self-report scale of positive and negative metacognitive beliefs about Body dysmorphic, thought-fusion and metacognitive control strategies. BDMCS comprises items relating to two sets of metacognitive beliefs in patients with BDD that are harmful to and not in control of cognitive functioning.

Table 2. Model fit indices for the model of the Iranian version of the BDMCS.

\begin{tabular}{cccccc}
\hline Model & N & GFI & AGFI & RMR & RMSEA \\
\hline & 600 & 0.99 & 0.99 & 0.004 & 0.001 \\
\hline
\end{tabular}

N: number of participants; GFI: Goodness of Fit Index; AGFI: Adjusted Goodness-of-Fit Index; RMR: Root Mean Square Residual; RMSEA: Standardized Root Mean Square Residual.

Table 3. Correlation results of Body Dysmorphic Metacognition Scale (BDMCS) and its factors with Yale-Brown Obsessive Compulsive Scale modified for BDD (BDD-YBOCS) and TFI.

\begin{tabular}{ccccccc}
\hline Variable & BDMCS & Factor 1 & Factor 2 & Factor 3 & Factor 4 & 0.47 \\
\hline (BDD-YBOCS) & 0.44 & 0.46 & 0.40 & 0.51 & 0.40 \\
TFI & 0.52 & 0.50 & 0.40 & 0.07 & 0.001 \\
Significance & 0.001 & 0.001 & 0.001 & 0.001 \\
\hline
\end{tabular}


Table 4. Reliability coefficient (of Cronbach's Alpha) of BDMCS and its factors.

\begin{tabular}{cccc}
\hline Factors & Sample size & No. of items & Cronbach's alpha \\
\hline Factor 1 & 600 & 5 & 0.90 \\
Factor 2 & 600 & 5 & 0.83 \\
Factor 3 & 600 & 5 & 0.81 \\
Factor 4 & 600 & 5 & 0.91 \\
Factor 5 & 600 & 5 & 0.88 \\
Total & 600 & 25 & 0.94 \\
\hline
\end{tabular}

Overall, this new BDMCS showed good psychometric properties. In addition, it exhibited strong face and content validity, convergent validity with constructs in the body dysmorphic disorder. Nevertheless, the present findings are preliminary, and future research should continue to examine the psychometric robustness of the BDMCS.

Findings of this study revealed that the BDMCS has a clear five-factor structure, congruent with its theoretical conceptualization. This is congruent with the MCT theory of Wells [8] [9] and studies about the efficacy of MCT on body dysmorphic disorder (e.g. [6]).

The higher positive correlation and significance of this scale and its factors with Yale-Brown Obsessive Compulsive Scale modified for BDD represents good convergent validity of this scale. The scale possesses high internal consistency, and the magnitude of the inter-item correlations is suggestive of a low to moderate overlap indicating low item redundancy. Internal consistency coefficient of 0.94 for the total scale and the coefficients of 0.81 to 0.91 for subscales indicate validity and high internal consistency of this scale.

BDMCS factors are significantly and positively associated with BDD symptoms and TFI.

Researchers have consistently found that BDD is characterized by a heightened subjective experience of worry as an uncontrollable process and any efforts at control prove futile and unproductive. Despite their acknowledged inability to control worry, it is interesting that individuals with BDD are highly invested in continuing with their efforts toward gaining control over worry and unwanted repetitive thoughts [6] [12] [13].

Finally, in case of relapse of BDD behavior, it may be helpful to derive and illustrate the role of metacognitive beliefs about BDD in the given episode together with other relevant cognitive-behavioral constructs.

The present results are preliminary in nature. Clearly future studies are required to further establish the psychometric properties of BDMCS. In particular, it would be necessary to determine the structure and reliability over time and with other samples. In addition, studies are required to examine the sensitivity of scale to treatment effects and recovery, if the scales are proven to be useful treatment evaluation tools. Longitudinal studies can be designed to evaluate the role of high levels of positive metacognitive beliefs in predisposing individuals to engage in BDD, and of negative metacognitive beliefs in maintaining problematic BDD behaviors.

Results of this study must be considered with regard to design limitations. Self-report biases, context effects and poor recall may have contributed to errors in self-report measurement. However, despite these limitations, we believe that this instrument may already be useful for eliciting metacognitive control strategies, thoughtfusion, positive and negative metacognitive beliefs about cognitive harm and uncontrollability and positive metacognitive beliefs about emotional self-regulation in clinical assessment and case formulation, and in providing a further step towards the development of a metacognitive conceptualization of BDD problem.

\section{Acknowledgements}

The authors would like to acknowledge of Professor Adrian Wells. We got the idea of his and Colleagues results about metacognitive therapy. The authors would also like to acknowledge the assistance of participants and all of the clinicians and research assistants who conducted the assessments.

\section{Authors' Contributions}

All authors had role in design, work, statistical analysis and manuscript writing. 


\section{Conflict of Interest}

The authors declare no conflict of interest.

\section{References}

[1] Van Ameringen, M., Patterson, B. and Simpson, W. (2014) DSM-5 Obsessive-Compulsive and Related Disorders: Clinical Implications of New Criteria. Depression and Anxiety, 31, 487-493. http://dx.doi.org/10.1002/da.22259

[2] Goodman, W.K. (2014) Obsessive Compulsive and Related Disorders. Psychiatric Clinics of North America, 37, xi-xii. http://dx.doi.org/10.1016/j.psc.2014.06.005

[3] Rabiei, M., Donyavi, V., Nikfarjam, M. and Nezhady, M.A.M. (2015) Validation of a Classification System for Obsessive-Compulsive and Related Disorders Based on DSM-5. Open Journal of Psychiatry, 5, 137-143. http://dx.doi.org/10.4236/ojpsych.2015.52015

[4] Vulink, N.C., Sigurdsson, V., Kon, M., Bruijnzeel-Koomen, C.A., Westenberg, H.G. and Denys, D. (2006) Stoornis in de lichaamsbeleving bij 3-8\% van de patienten op de poliklinieken Dermatologie en Plastische Chirurgie [Body Dysmorphic Disorder in 3-8\% of Patients in Outpatient Dermatology and Plastic Surgery Clinics]. Nederlands Tijdschrift voor Geneeskunde, 150, 97-100.

[5] Williams, J., Hadjistavropoulos, T. and Sharpe, D. (2006) A Meta-Analysis of Psychological and Pharmacological Treatments for Body Dysmorphic Disorder. Behaviour Research and Therapy, 44, 99-111. http://dx.doi.org/10.1016/j.brat.2004.12.006

[6] Rabiei, M., Mulkens, S., Kalantari, M., Molavi, H. and Bahrami, F. (2012) Metacognitive Therapy for Body Dysmorphic Disorder Patients in Iran: Acceptability and Proof of Concept. Journal of Behavior Therapy and Experimental Psychiatry, 43, 724-729. http://dx.doi.org/10.1016/j.jbtep.2011.09.013

[7] Donyavi, V., Rabiei, M., Nikfarjam, M. and Nezhady, B. (2015) Body Image and Meta-Worry as Mediators of Body Dysmorphic Disorder. International Journal of Clinical Medicine, 6, 170-176. http://dx.doi.org/10.4236/ijcm.2015.63022

[8] Wells, A. (2000) Emotional Disorders and Metacognition: Innovative Cognitive Therapy. John Wiley \& Sons, Chichester, New York, ix, 236 p.

[9] Wells, A. and Matthews, G. (1996) Modelling Cognition in Emotional Disorder: The S-REF Model. Behaviour Research and Therapy, 34, 881-888.

[10] Papageorgiou, C. and Wells, A. (2003) An Empirical Test of a Clinical Metacognitive Model of Rumination and Depression. Cognitive Therapy and Research, 27, 261-273.

[11] Cooper, M. (2003) Emotional Disorders and Metacognition: Innovative Cognitive Therapy. British Journal of Clinical Psychology, 42, 105-106.

[12] Rabiei, M., Salahian, A., Bahrami, F. and Palahang, H. (2011) Construction and Standardization of the Body Dysmorphic Metacognition Questionnaire. The Journal of Mazandaran University of Medical Sciences, 21, 43-52.

[13] Cooper, M. and Osman, S. (2007) Metacognition in Body Dysmorphic Disorder-A Preliminary Exploration. Journal of Cognitive Psychotherapy, 21, 148-155. http://dx.doi.org/10.1891/088983907780851568

[14] Veale, D. (2004) Advances in a Cognitive Behavioural Model of Body Dysmorphic Disorder. Body Image, 1, $113-125$. http://dx.doi.org/10.1016/S1740-1445(03)00009-3

[15] Hollander, E. and Benzaquen, S.D. (1997) The Obsessive-Compulsive Spectrum Disorders. International Review of Psychiatry, 9, 99-109.

[16] Phillips, K.A., Hollander, E., Rasmussen, S.A., Aronowitz, B.R., DeCaria, C. and Goodman, W.K. (1997) A Severity Rating Scale for Body Dysmorphic Disorder: Development, Reliability, and Validity of a Modified Version of the Yale-Brown Obsessive Compulsive Scale. Psychopharmacology Bulletin, 33, 17-22.

[17] Rabiei, M., Khormdel, K., Kalantari, K. and Molavi, H. (2010) Validity of the Yale-Brown Obsessive Compulsive Scale Modified for Body Dysmorphic Disorder (BDD) in Students of the University of Isfahan. Iranian Journal of Psychiatry and Clinical Psychology, 15, 343-350.

[18] Wells, A., Gwilliam, P. and Cartwright-Hatton, S. (2001) The Thought Fusion Instrument (Unpublished Self-Report Scale). Unpublished Scale Manchester, University of Manchester, Manchester.

[19] Khoramdel, K., Rabiei, M., Molavi, H. and Neshatdoost, H.T. (2010) Psychometric Properties of Thought Fusion Instrument (TFI) in Students. Iranian Journal of Psychiatry and Clinical Psychology, 16, 74-78.

[20] Nunnally, J. and Bernstein, I. (1994) Psychometric Theory. 3rd Edition, McGraw-Hill, New York.

[21] Floyd, F.J. and Widaman, K.F. (1995) Factor Analysis in the Development and Refinement of Clinical Assessment Instruments. Psychological Assessment, 7, 286-299. http://dx.doi.org/10.1037/1040-3590.7.3.286 